\title{
Radical Guide Vane Design and Optimization
}

\author{
Max E. Rife, Fernando Barbarossa † Luca di Mare $\ddagger$ and Anthony B. Parry $\S$
}

Vibration UTC, Department of Mechanical Engineering, Imperial College London, London, SW7 2BX, UK

Jeffrey S. Green ฯ

Rolls Royce plc, Derby, DE24 8BJ, UK

\begin{abstract}
Turbofan engines are connected to the airframe by a pylon which crosses the bypass duct downstream of the fan. The presence of the pylon generates a potential field that influences the upstream flowfield. Distortion generated by the pylon can be detrimental to the fan stability and integrity and as such must be controlled. This work presents a new method for the two-dimensional design of fan outlet guide vane cascades in the presence of the potential flow distortion caused by the pylon. The method, based on surface singularities, is capable of mitigating the distortion while preserving the performance of the nominal cascade. Design targets are achieved by manipulating both airfoil geometry and position within the cascade. As the distortions caused by the pylon
\end{abstract}

\footnotetext{
${ }^{*}$ Research Associate, Email: m.rife@imperial.ac.uk

${ }^{\dagger} \mathrm{PhD}$ Student, Email: fernando.barbarossa12@imperial.ac.uk

${ }^{\ddagger}$ Lecturer, Email: 1.di.mare@imperial.ac.uk

$\S$ Visiting Academic, Email: a.parry@imperial.ac.uk

『Engineering Associate Fellow - Vibrations, Email: jeffrey.green@rolls-royce.com
} 
obey essentially inviscid fluid mechanics, the variation in load and loss parameters across the cascade can be approximated surprisingly well with a singularity method. The design rational of controlling the incompressible load distribution leads to cascades with uniform load and loss even when viscous and compressible effects are considered using high fidelity computational fluid dynamics. The new approach provides a significant speed up over methods based on computational fluid dynamics and is valuable as an initial design tool. 


\section{Nomenclature}

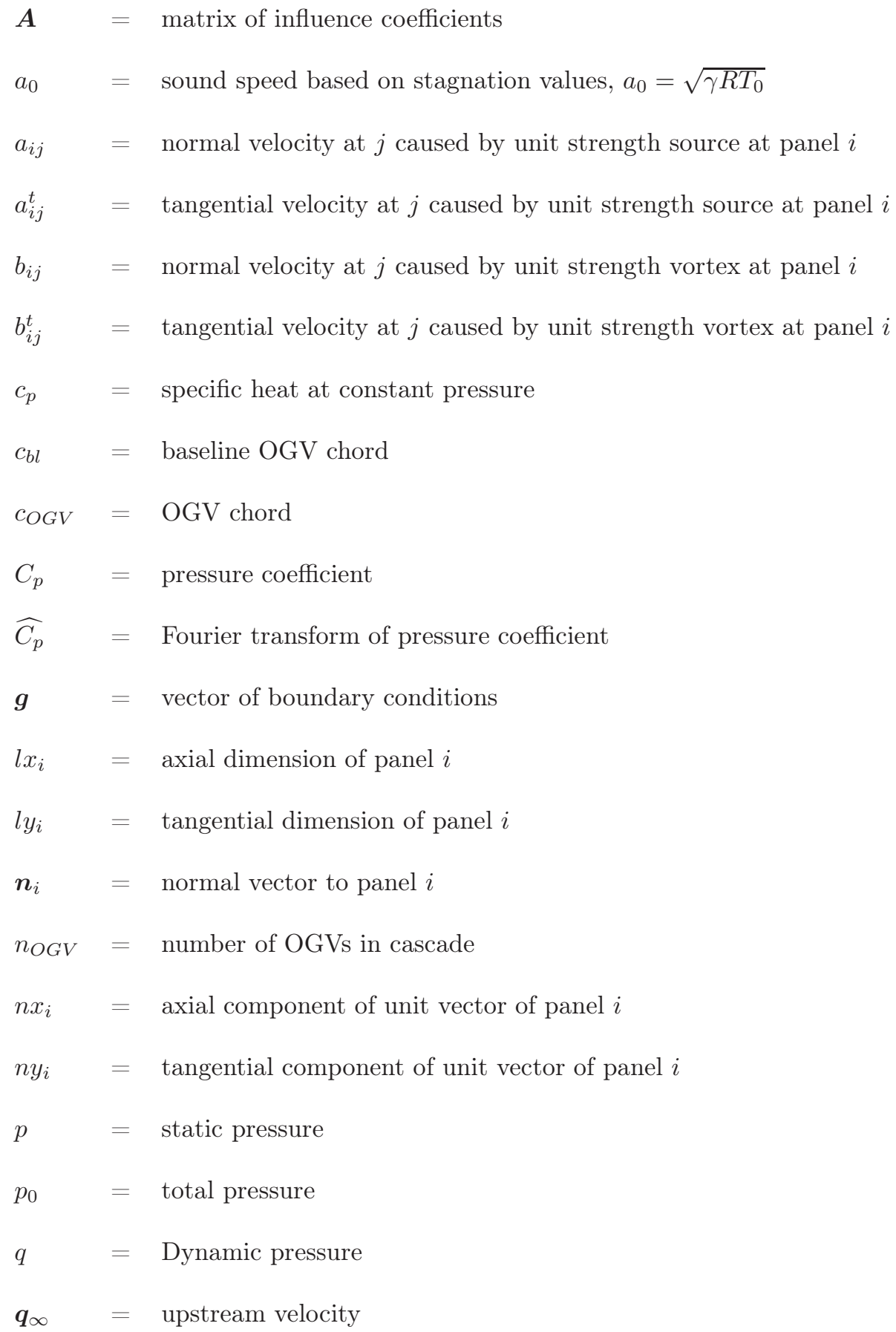




$$
\begin{array}{ll}
R & =\text { universal gas constant } \\
s & =\text { curvilinear coordinate } \\
T & =\text { static temperature } \\
T_{0} & =\text { total temperature } \\
U_{\infty} & =\text { Upstream axial velocity component } \\
V_{\infty} & =\text { Upstream tangential velocity component } \\
\boldsymbol{w}(\boldsymbol{z}) & =\text { complex velocity at location } \boldsymbol{z} \\
\boldsymbol{z} & =\text { location within complex plane } \\
z_{0} & =\text { vortex location } \\
\gamma & =\text { ratio of specific heats } \\
\gamma & =\text { vortex strength } \\
\gamma^{\prime} & =\text { scaled vortex strength } \\
\widehat{\gamma^{\prime}} & =\text { Chebyshev transform of scaled vortex strength } \\
\gamma & =\text { vector of vortex and source strengths } \\
\mu & =\text { Mach number based on stagnation values } \\
\gamma_{0} & =\frac{T}{T_{0}} \\
\gamma_{0} & =
\end{array}
$$

\section{Introduction}

It has long been known that bodies downstream of a stator cascade generate a potential field that propagates upstream through the cascade of stators, often leading to significant circumferential variation of static pressure which 
is detrimental to the upstream rotor, the so-called rotor-stator-strut problem. The effect was first documented by Hemsworth [1] in a review of the lessons learned in the development of the TF39 high bypass ratio engine. In order to reduce the once per revolution vibration of the fan blades caused by the pressure field of the pylon, the pylon was integrated into the guide vane cascade and 5 different blade geometries were used in the cascade.

After the rotor-stator-strut problem was identified, numerical efforts to minimize the effect began with potential models, such as in the work of Rubbert et al. [2], Barber \& Weingold [3], Yokoi et al. [4], and Cerri \& O'Brien [5], to name a few. Of course, experimental work was performed concurrently, such as that of O'Brien et al. [6] and Ng et al. [7]. The agreement between the potential models and the experimental data was generally very good.

Rubbert et al. [2] conducted perhaps the first study of the stator-strut problem. The work demonstrated the long-reaching potential effects of the strut in the azimuthal sense, affecting flow direction a quarter of the way around the duct from the strut by as much as 2 degrees. Attempts were made by Rubbert et al. [2] in two dimensions to minimize the upstream pressure distortion by providing a uniform mass flux distribution through the stator cascade. The result of the optimization was a unique camber line geometry for each blade within the cascade that provided a given vorticity distribution based on equal axial mass flux in the middle of each passage. The resulting flowfield ahead of the stators was said to be uniform. While the vorticity distribution shape was to be similar for each blade, the amplitude was not specified. As a result, the two blades nearest the strut had noticeably different pressure distributions. As such one blade was more highly loaded than the other, though it was noted that the increase in loading occurred primarily on the pressure side, while the suction side pressure distributions were nearly identical for the two blades, so neither was more prone to boundary layer separation than the other blade.

Barber and Weingold [3] used a Douglas-Neumann potential method to predict the flowfield through a twodimensional cascades. The work studied a cascade of guide vanes ahead of a cascade of struts, as well as a combined cascade of guide vanes and struts. Validated using experimental data from linear cascades matching the numerical geometries, the numerical model was shown to provide accurate results for both the tandem cascades and integrated 
configurations.

Yokoi et al. [4] performed an experimental study on the effect of stator setting angle patterns on the upstream pressure field and rotor vibration. It was shown that a non-uniform stator setting angle could effectively reduce the upstream pressure distortion and, subsequently, the rotor vibration. A simple calculation based on the continuity equation was used to predict the upstream pressure distribution, which agreed well with the experimental results. The good agreement between the experimental and analytical results demonstrates the dominant role that the potential field plays in the rotor-stator-strut problem.

Using a potential flow model, Cerri \& O'Brien [5] studied the effects of blade stagger angle on the pressure distribution upstream of a stator-strut system. After an optimization campaign which modified the blade stagger angles and strut azimuthal location, the upstream pressure distribution was found to be significantly more uniform than the baseline case. The optimization strategy was to modify only stagger angle and strut azimuthal location so as to avoid the costs associated with design and manufacture of blades with various camber geometries. In order to ensure sensible results, the change in stagger angle was limited to $\pm 4^{\circ}$. In fact, the optimized cascades varied the stagger angles by no more than $\pm 3^{\circ}$ and it was suggested that such a narrow range of re-staggering would only slightly affect blade performance.

O'Brien et al. [6] performed an experimental investigation of the unsteady pressure disturbances at the rotor caused by downstream struts. The experimental results showed that the effect of the struts was reduced by increasing the axial separation between the struts and the rotor. Additionally, a computational model of the cascade using the Douglas-Neumann singularity superposition method was shown to yield accurate results compared to the experimental data. While no attempt at modifying the cascade geometry was made by O'Brien et al., the work demonstrates clearly the decaying nature of the influence of the strut on the rotor and the accuracy of a potential method in the analysis of this type of problem.

$\mathrm{Ng}$ et al. [7] studied experimentally the influence of the unsteady pressure field of downstream struts on rotor 
blades. The work investigated the influence of strut spacing on the unsteady response of the rotor. The influence of the downstream strut was found to be larger than that of stator row. An exponential decay of the pressure distortion caused by the strut was identified, which agreed with the analytical model of O'Brien et al. [8].

It should also be noted that the presence of downstream bifurcations such as pylons or struts are associated with rotor noise issues too. Holewa et al. [9] performed a numerical study on the noise generation of a two-dimensional rotor-stator-strut problem. The presence of the struts were found to affect the system noise. From analysis of unsteady forces the stator vanes, struts, and bifurcations were identified as the dominant sound sources.

Optimization efforts with respect to system noise in the view of the rotor-stator-strut problem have been performed, for example by Giacché et al. [10]. While noise effects are important to consider in the design of a cascade, the current work focuses only on the steady flowfield and as such noise generation and mitigation is not considered here.

During the mid-1980s Kodama [11] demonstrated the first use of an actuator disk method for the prediction of the performance of a stator cascade subject to downstream static pressure distortions. Comparing the results of the actuator disk method with experimental data provided good agreement. Kodama \& Nagano [12] later went on to further validate the actuator disk model with experimental data. Additionally, the authors presented a method for directly calculating the required stator exit flow angle to mask the effect of downstream bodies. Using this method a modified experimental model of the guide vane design was tested. A noticeable reduction in the upstream pressure distortion was found with the modified vanes compared to a uniform stator cascade.

Parry [13] used a tandem fan-OGV actuator disk method to evaluate the upstream flow disturbance and optimize the OGV design. The tandem actuator disk method demonstrated a back-effect of the fan that reduced the pressure disturbance caused by downstream struts compared to the results without the fan. These results contrast with the findings of Cerri et al. [14] but were in agreement with experimental data. The optimization procedure aimed to minimize the peak-to-peak fluctuation of the Mach number variation at the OGV leading edge by re-cambering the 
OGVs in up to 5 groups of blade types. The perturbations at the OGV leading edge were shown to be reduced by up to $50 \%$ using 3 blades and up to $75 \%$ with 5 camber profiles.

The work of Parry [13] was later extended by Parry \& Bailey [15] where a rotor-stator-strut-pylon configuration was considered using a tandem actuator disk model. The actuator disk model was validated against experimental data and showed very good agreement. While stator geometry optimization was not considered by Parry \& Bailey, it was shown that aligning the struts with the streamlines of the pylon-only flowfield reduced the flowfield disturbances to the levels of the pylon alone.

Around the same time as the works of Parry and Parry et al. were being published Chiang \& Turner [16] published one of the first uses of computational fluid dynamics (CFD) in the study of the rotor-stator-strut problem. Using a two-dimensional solver, Chiang \& Turner showed that a uniform stator cascade could significantly amplify the pressure distortion caused by the strut. Additionally, a modified cascade with guide vanes re-staggered such that the exit flow angle would match a predicted 'optimum' was evaluated. The results of the modified cascade demonstrated a significant reduction in the upstream pressure distortion and a more uniform loading of the guide vanes from blade to blade.

Since the work of Chiang \& Turner most subsequent numerical studies of the rotor-stator-strut problem have been conducted using high fidelity CFD tools. Shahpar et al. [17] was possibly the first use of three-dimensional CFD for the optimization of a bypass guide vane cascade. Using Reynolds Averaged Navier Stokes (RANS), the authors performed an optimization where all blades in the cascade were re-staggered independently; the upstream pressure variation approximately one chord upstream of the guide vane cascade was be reduced by $78 \%$. However, the resulting geometry led to adjacent blades having large differences in stagger angle, with variations of up to six degrees between adjacent blades. This stagger angle scheme resulted in an increase in total pressure loss for some OGVs of almost 40\%. Shahpar et al. provides a good example of the limitations of an optimization strategy that targets only the upstream distortion: by optimizing only for upstream distortion the resulting geometry may 
negatively impact the guide vane cascade performance as non-uniform load around the cascade may be introduced. This was identified by Shahpar et al. and multi-objective optimizations were conducted that yielded less aggressive re-staggering schemes with reductions to both upstream distortion and cascade pressure loss.

Enoki et al. [18] used a three-dimensional unsteady CFD solver to investigate the effect of pylon spacing on the fan forced vibration. The work tested two different configurations of axial distance between the stator cascade and the pylon. Good agreement with experimental results was found. Additionally, a two-dimensional actuator disk model was compared to the experimental data, with good agreement.

While viscous CFD methods may have become the state of the art for the study of the rotor-stator-strut problem and the optimization of a guide vane cascade, they are not without drawbacks. Most significant of these is the computational cost associated with high fidelity CFD. As an example, Shahpar et al. [17] noted that an optimization procedure could take between 2 and 7 days to complete when using between 6 and 13 workstations in parallel. Long run times limit the value of such a tool for early design work, where many designs may need to be generated and evaluated quickly. As such, the potential model has experienced a recent revival as the tool of choice for some researchers when tackling the rotor-stator-strut problem.

Using a potential method, Rife et al. [19, 20] demonstrated the value of an optimization method based on targeting uniform blade-to-blade loading around the cascade. These works were able to generate geometries that could significantly mask the upstream propagating pressure distortion caused by a downstream body while satisfying the target for uniform blade-to-blade loading in the guide vane cascade. Additionally, it was shown that indicators of loss through the cascade, such as incidence variation or the value of peak suction surface velocity, were minimized as a result of the optimization, without explicitly being included in the objective functions.

Building on the findings of Rife et al. the goals of this paper are threefold. First, playing to the strengths of the potential method, an optimization approach that is based on the solution of a least squares problem is presented. The benefits of this approach will be discussed, most notably the significantly reduced time taken during optimization. 
Secondly, the geometric freedom of the approach allows for non-conventional cascade geometries, with variation to axial and tangential location available as a degree of freedom within the optimizer. The value of allowing the solid body translation of the blades will be discussed. Thirdly, the accuracy of the results from the potential model will be assessed against high fidelity CFD. Good agreement between the simulations demonstrates that the potential method is a useful tool for the design and optimization of a guide vane cascade. The speed at which the optimized cascade can be obtained, while retaining good accuracy of the result, enhances the value of the approach in an early design stage.

Towards these goals the paper will begin with a review of the methodology used in the solution of the flowfield and the approach to optimization. Results will then be presented for the optimization of a two-dimensional guide vane cascade ahead of a single pylon. The optimized geometry will then be evaluated using CFD to validate the design and demonstrate the robustness of the approach. Finally, conclusions from the work will be presented.

\section{Methodology}

\subsection{Basic flowfield solver}

The outlet guide vanes and pylon are modeled in two dimensions so the row of guide vanes becomes a twodimensional cascade. The flowfield through the guide vane cascade is evaluated using a tool based on the DouglasNeumann singularity superposition method. As the guide vanes generally have very thin sections compared to the amount of camber, the guide vanes are modeled by their camber lines. Each blade is discretized and represented by a series of straight panels. On each panel a point vortex is located at one quarter panel chord location and a control point is located at three quarters panel chord location. Thick bodies, such as the pylon, cannot be accurately represented

by their camber lines and thickness must be included. The boundaries of such thick entities are discretized using straight lines. Each panel then has constant source and constant vorticity. A system of equations can be constructed 
with the no-permeability and Kutta conditions as boundary conditions. Solution of the system of equations yields the singularity strength for each element. A compressibility correction similar to the Prandtl-Glauert transformation is employed. A more complete discussion of the flowfield solver is provided in the appendix.

\subsection{Linearization}

The system of equations for the flowfield through a cascade comprised of both thin and thick bodies can be generalized as

$$
A \gamma=b
$$

where $\boldsymbol{A}$ is the matrix of influence coefficients, $\gamma$ is the vector of singularity strengths, and $\boldsymbol{b}$ is the right hand side satisfying the no-permeability and Kutta conditions. The matrix of influence coefficients is known from the geometry, and the right hand side is known from the boundary conditions. Then, the singularity element strengths are obtained by inversion and solving for $\gamma$, i.e.

$$
\gamma=A^{-1} b
$$

The changes to the flowfield caused by a geometrical change to the cascade, $\boldsymbol{d x}$, can be evaluated as

$$
\frac{d A}{d x} \gamma+A \frac{d \gamma}{d x}=\frac{d b}{d x}
$$

the terms $\frac{d \boldsymbol{A}}{d x}, \gamma, \boldsymbol{A}$, and $\frac{d b}{d x}$ are all known or calculable from the starting and perturbed geometry. Final evaluation of the changes in element strengths, $\frac{d \gamma}{d x}$, is obtained from

$$
\frac{d \gamma}{d x}=A^{-1}\left(\frac{d b}{d x}-\frac{d A}{d x} \gamma\right)
$$


The value $\frac{d \gamma}{d x}$ expresses the change in singularity strengths throughout the cascade due to a known geometrical change, $\boldsymbol{d} \boldsymbol{x}$. The ability to evaluate this is derivative is central to the optimization strategy employed in this work.

\subsection{Optimization}

The optimization method used in the current work aims to tackle guide vane inverse design using a non-iterative approach. A starting baseline cascade is first evaluated using the standard solver, and the values of the objective function components (costs) can be computed. Following the work of Rife et al. [19, 20], the objective function used is based on blade-to-blade loading variation, upstream pressure distortion, and pylon circulation. This approach has been shown to provide optimized geometries capable of fully suppressing any upstream pressure distortion while minimizing losses through the cascade.

Blade loading is evaluated as a Chebyshev transform of the scaled circulation along the chord. The pressure coefficient could be used by approximating the blades by their camber lines, but a strong singularity then exists at the leading edge that must be removed to reduce the number of terms required to accurately represent the distribution along the chord. The scaled circulation, $\gamma^{\prime}$, is defined as:

$$
\gamma^{\prime}=\gamma \sqrt{x-x_{l e}}
$$

where $\gamma$ is the vortex strength, $x$ is the axial position of the vortex, $x_{l e}$ is the axial location of the leading edge of the blade. The scaled distribution varies smoothly across the length of the chord and singular values are avoided. Consequently, reconstruction with relatively few Chebyshev coefficients is possible.

For the upstream pressure distribution around the cascade, Fourier coefficients of the pressure coefficient one chord upstream of the baseline cascade leading edge are used.

The target values of the objective function components must be known before optimization. These are obtained as the difference between the values of the cost function components for the baseline cascade with and without any 
downstream bodies. The target cost values are stored in a target vector, $\boldsymbol{d y}$.

The final objective function can be written as:

$$
\sum_{i=1}^{n_{O G V}} \sum_{j=0}^{j_{\max }}\left({\widehat{\gamma^{\prime}}}^{j}{ }_{i}^{j}-\left({\widehat{\gamma^{\prime}}}_{t g t}\right)_{i}^{j}+\sum_{k=1}^{k_{\max }}\left(\widehat{C_{p}}\right)^{k}-\left(\widehat{C_{p}}\right)_{t g t}^{k}=0\right.
$$

Where $\left(\widehat{\gamma}^{\prime}\right)_{i}^{j}$ is the $j^{\text {th }}$ Chebyshev coefficient of the scaled circulation on blade $i$ and $\left(\widehat{C}_{p}\right)^{k}$ is the $k^{t h}$ Fourier coefficient of the upstream pressure distribution. The subscript $t g t$ indicates target values and $n_{O G V}$ is the number of OGVs in the cascade. $j_{\max }$ and $k_{\max }$ are respectively the maximum number of Chebyshev and Fourier components to be included in the optimization.

During optimization a series of known geometrical changes are applied to the cascade. In the current work the changes available are camber line modifications and solid body translations in both the axial and tangential directions. For the camber line changes a series of orthogonal polynomials are used to describe displacement in the direction normal to the unperturbed camber line, as shown in Fig. 1. Solid body translation is achieved by using Fourier harmonics to translate the blades in the cascade in the axial or tangential direction, as demonstrated in Fig. 2 .

During the optimization step, each change is applied independently and the linearized changes to the cost function components are evaluated and stored into a Jacobian matrix, $\boldsymbol{J}$. The aim of the optimization is to apply a combination of geometrical changes that will result in the desired changes in the cost components stored in $\boldsymbol{d} \boldsymbol{y}$ being applied. The problem can be written simply as

$$
J d x=d y
$$

where $\boldsymbol{d} \boldsymbol{x}$ represents the unknown geometry changes required to achieve values of the costs that are equivalent to those of the baseline cascade in isolation. The geometry changes are obtained by inversion of the Jacobian matrix and multiplication with the target vector, i.e. 


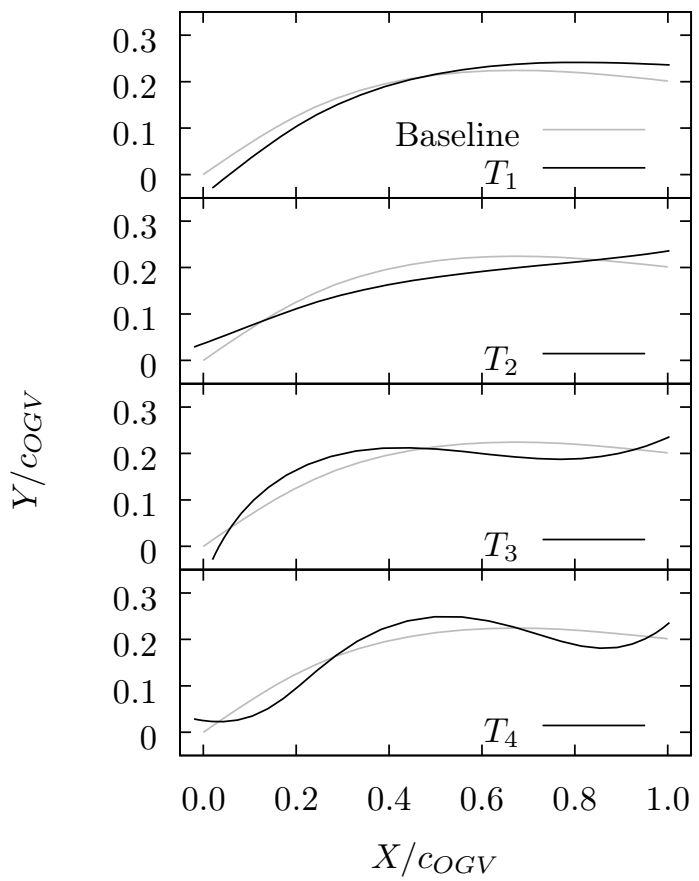

Figure 1: Camber line displacement patterns for various Chebyshev polynomials.

$$
d x=J^{-1} d y
$$

This turns the optimization into a least squares problem, which can be solved in one computational step. The result is the vector of required geometrical changes to be applied to the cascade geometry in order to achieve the target cost values.

As the optimization is based on a linearized approximation, the resulting geometry displacement may be slightly off from the true solution. As a result, performing a subsequent optimization on the resulting geometry helps to fully satisfy the objective function. Typically, three or four optimizations are sufficient to satisfy the objective function, and further optimizations result in negligible changes to the geometry.

All optimizations in this work were performed on a workstation equipped with an Intel@ Core $^{\mathrm{TM}} \mathrm{i} 3-4340$ processor 


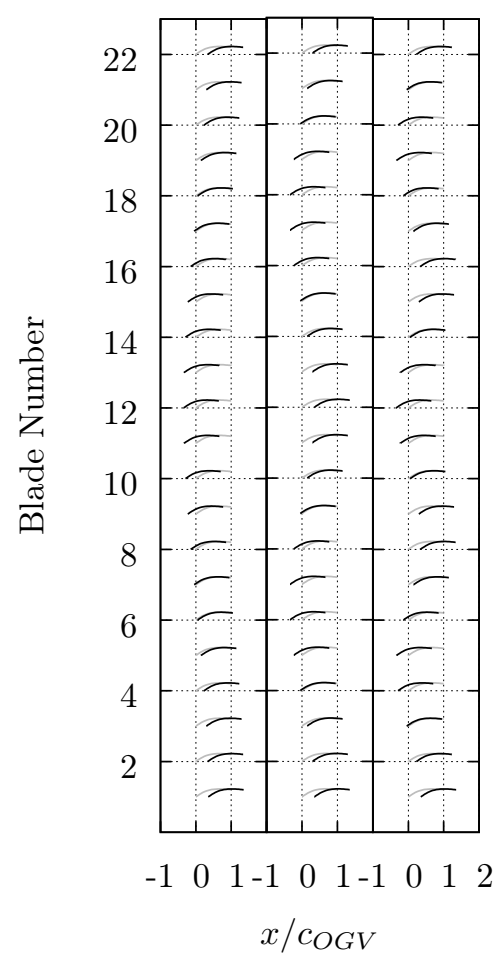

Figure 2: Fourier harmonics of axial solid body translation of guide vane cascade. Fourier harmonics range from 1 to 3 , increasing from left to right.

running at $3.6 \mathrm{GHz}$ with $32 \mathrm{~GB}$ of RAM.

\section{Results}

To demonstrate the new approach to cascade optimization, a cascade of 22 guide vanes has been optimized. This section will comprise of two parts. The first part will present the results of a series of increasingly complex optimizations. The objective of this study is to evaluate the effect of the solid body translation on the satisfaction of 
the optimization goals. The second part will provide a validation of the potential method against high fidelity CFD results. Results from the baseline and an optimized cascade will be compared between the methods.

For all optimizations the blade camber line was modified with up to 5 Chebyshev coefficients, and 5 Fourier coefficients were used to translate the blades in the axial and tangential directions. The optimization process targeted minimization of 5 Chebyshev coefficients for the loading distribution of each blade and 9 Fourier coefficients of the upstream pressure distribution. These values were chosen as experience had demonstrated that they provide a good balance between runtime and optimization results. While not presented here for the sake of brevity, a study on the effect of the number of coefficients used to modify the geometry showed that the optimization would favour optimizing for blade loading at the expense of the upstream pressure distribution. This is likely due to the larger number of blade loading terms in the target vector compared to the number of terms related to the upstream pressure distribution.

\subsection{Effect of solid body translation}

The approach to optimization described above includes the freedom to translate the guide vanes in both the tangential and axial directions. In order to assess the value of this solid body translation a series of increasingly complex optimizations are compared. Table 1 identifies the cases and the degrees of freedom used in the optimization. The CS, CSA, and CST cases were run three times, and the CSAT case was run four times. The optimizing tool only allows for translation of the blades in one direction during an optimization, and the order of translations for the CSAT case was tangential for the first run, axial for the second, then tangential followed by axial for the third and fourth runs, respectively. The results shown here are for the final geometries of the optimizations.

To compare the results of the four different optimizations in Table 1 the optimized geometries are compared against the baseline geometry in Fig. 3. The top image in Fig. 3 shows the optimized geometry after modifying only camber and stagger angles. Below this is the optimized cascade with camber, stagger, and axial translation degrees 


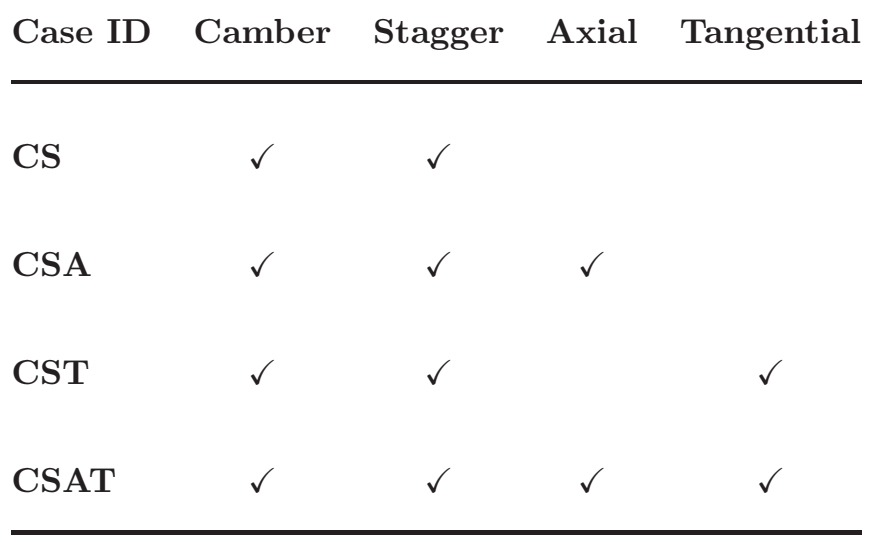

Table 1: Table of case IDs and geometry variables used in optimization.

of freedom. Blades 1-9 are moved axially forward (away from the pylon), and blades 12-20 are moved rearwards (toward the pylon). In the third image the optimized cascade with freedom of camber, stagger, and tangential location is presented. The optimized cascade exhibits an increased solidity for the portion of the cascade to the left of the pylon and a lower solidity to the right side of the pylon. It is also evident from this figure that the optimization has still made use of modifications to the camber and stagger angle; the blades to the left of the pylon clearly have an increased camber while blades to the right of the pylon have a noticeably reduced camber, compared to the baseline. The result of this re-cambering is that the blade trailing edges are no longer aligned in a nominally axial direction but appear to divert around the pylon. Finally, the bottom image in Fig. 3 shows the optimized cascade where both axial and tangential solid body translation has been employed. This optimized geometry exhibits traits of both the CSA and CST cases, with the solidity increased to the left of the pylon and the blades moved away from the pylon whilst on the right of the pylon the solidity is slightly reduced and the blades moved toward the pylon. Additionally, one can see the increased camber for blades 4-10 and reduced camber for blades 12-20, compared to the baseline geometry. As with the CST case, these modifications indicate an attempt to divert the flow around the pylon.

Figure 3 shows that the resulting geometry from the CSAT optimization has significantly more tangential translation applied to the cascade than axial translation. This is a result of performing a tangential translation in the first 


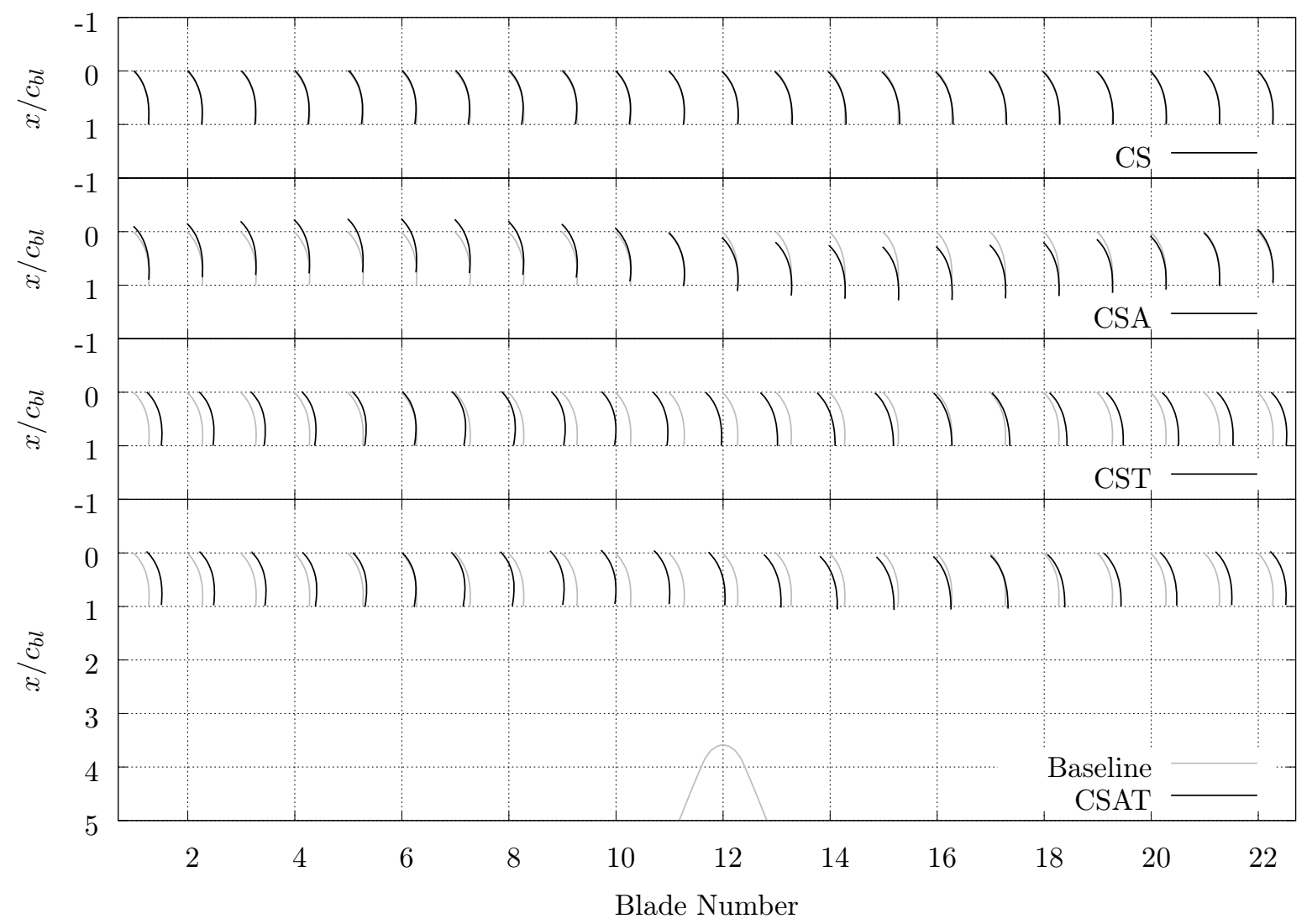

Figure 3: Comparison of baseline and optimized geometries with varying solid body translation degrees of freedom.

optimization pass, in which the most significant changes are applied. Had the first pass employed axial translation instead, the final geometry would show significantly more axial displacement than tangential.

To better visualize and understand the geometrical changes, the variation in stagger angle for all optimized cases is plotted in Fig. 4, with the baseline cascade plotted below for reference. A cyclical variation in the stagger angle is seen for all optimized cases, with blades having an optimized stagger angle that is generally less for blades 1-11 than for blades 13-22. In the CSA case, where axial translation is used in the optimization, the variation in the 


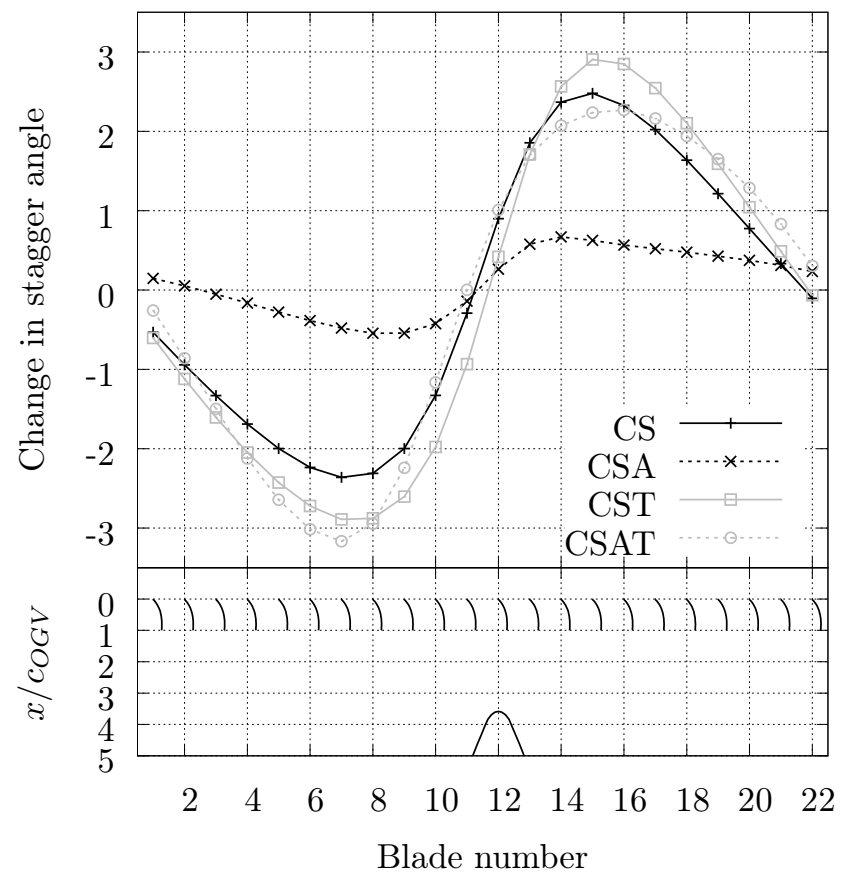

Figure 4: optimized stagger angle change.

stagger angle is noticeably less than for the other cases; the modification to the stagger angle is less than $\pm 1^{\circ}$ for all blades, while for all other cases up to approximately $\pm 3^{\circ}$ change in stagger angle is applied. In the CSA case the axial translation of the blades results in a local change to the plane of the cascade. This change to the inclination of the cascade has a similar effect to re-staggering the blades, and as such explains why the stagger angle change is smaller for the CSA case than all other cases.

The change to the camber line geometry from each of the optimizations is presented in Fig. 5. Each of the cases demonstrates a similar trend in the re-cambering strategy: increased camber for blades 3-11 and reduced camber for blades 12-21. However, it can be noted that for cases CS and CSA the amount of re-camber applied to blades 1-6 is noticeably less than that used in cases CST and CSAT. Nevertheless, the general trend in the geometry modifications 


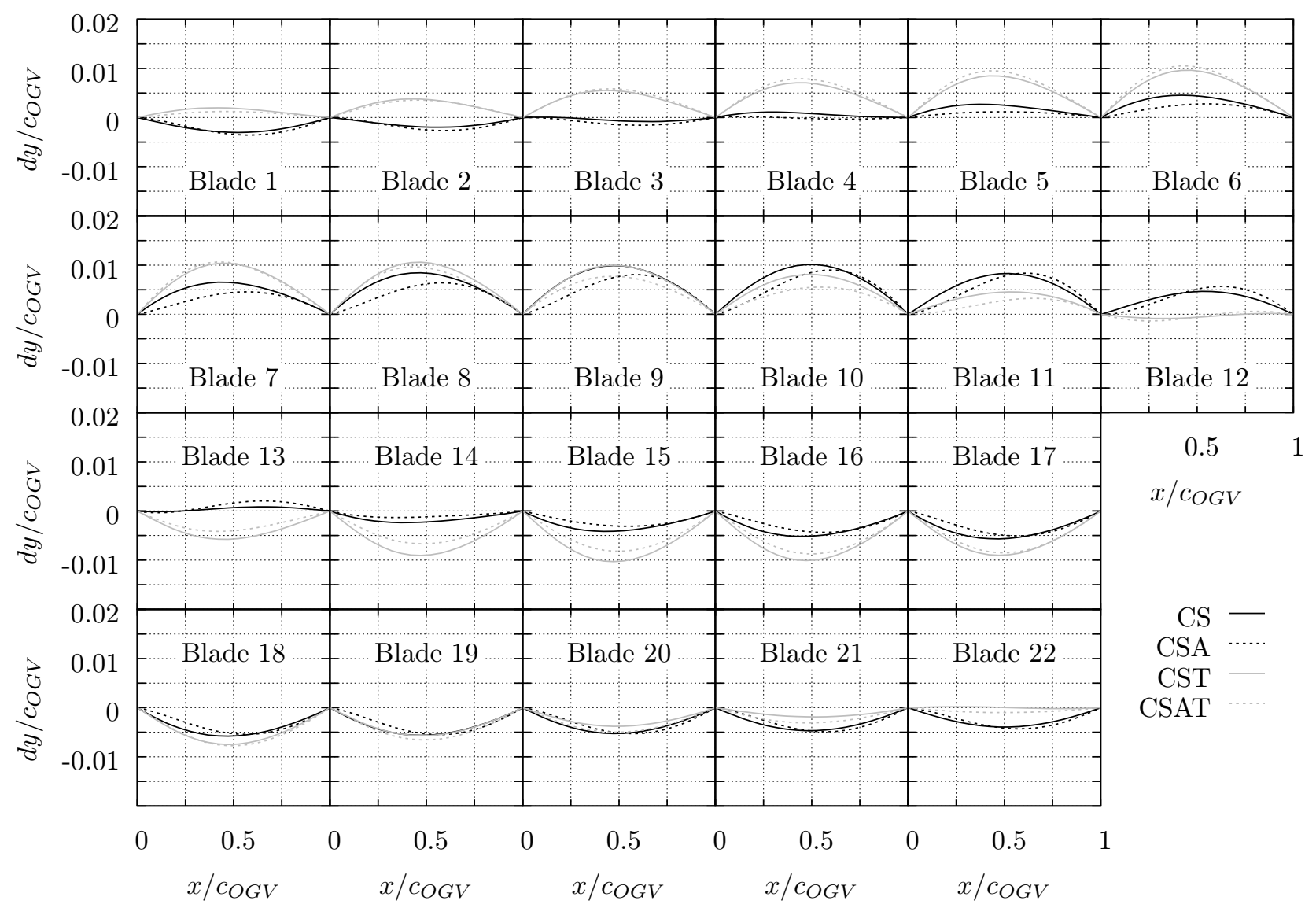

Figure 5: Change in blade camber line geometry, normalized by blade chord.

for all cases of optimization is, as noted above, to divert the flow around the pylon.

Having evaluated the changes to the geometries resulting from the optimizations, attention is now turned to the changes in the flowfield. Figure 6 plots the pressure coefficient at an axial location of $x / c_{b l}=-1$, one chord upstream of the baseline cascade leading edge location. The uniform cascade results in a large variation in the upstream pressure coefficient around the cascade, with an amplitude of approximately 0.1. By modifying only the 


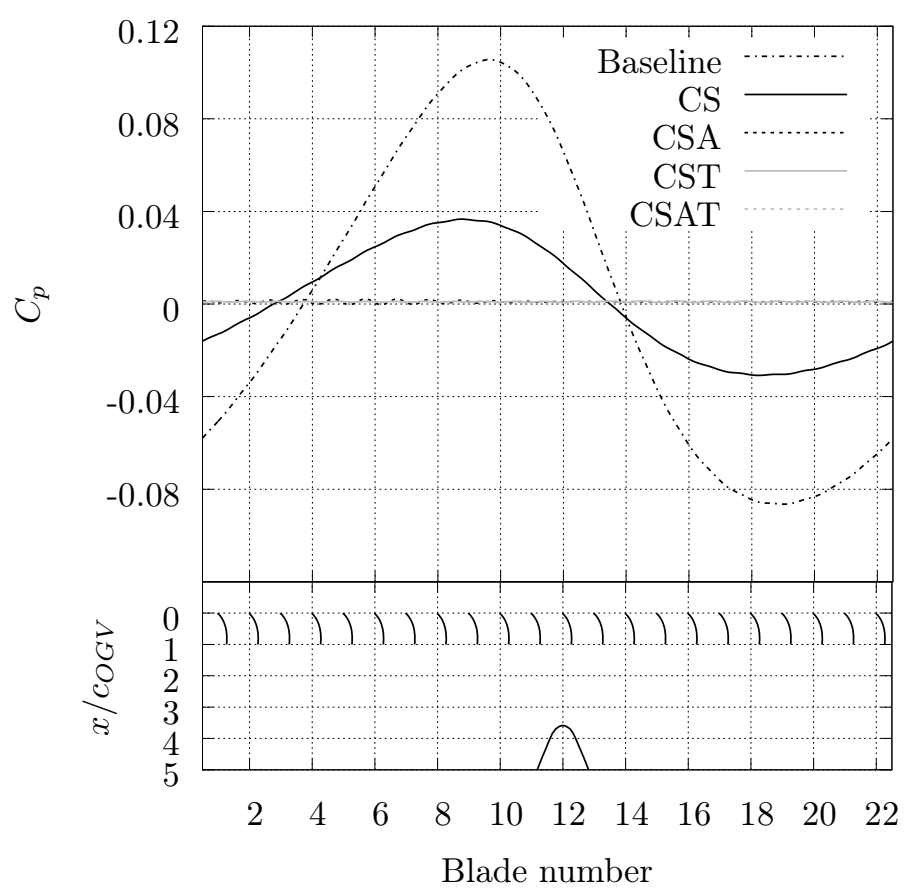

Figure 6: Pressure coefficient distribution for axial location one chord upstream of baseline cascade leading edge.

blade camber and stagger angle, case CS, this distortion is reduced to an amplitude of less than 0.04. However, by allowing freedom in either the axial or tangential direction, or both in the CSAT case, the upstream pressure distortion is seen to be practically eliminated.

The optimization goals are not only to reduce the upstream pressure distortion, but also to arrive at a uniform blade-to-blade loading variation around the cascade so that all blades are loaded similarly. To evaluate the effectiveness of this, the loading distribution for all 22 blades in each cascade is plotted in Fig. 7. The loading is given as the difference between the the pressure coefficient on the pressure and suction sides of the blade. The baseline cascade is shown in the top left of the figure and there is clear variation in the loading of the blades around the cascade. However, for all optimized cases, the loading variation is minimized, and very closely matches that of the 


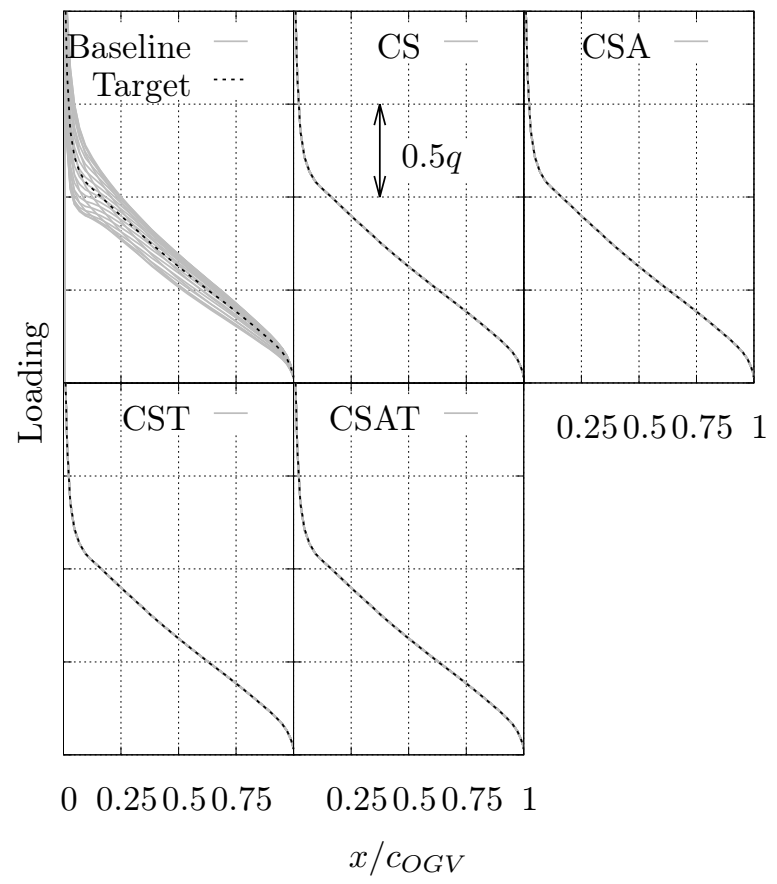

Figure 7: Loading variation for all blades in cascade, compared to target loading represented by dashed black line. target distribution, designated by the dashed black line.

In summary, the results presented above demonstrate the effectiveness of various geometrical changes towards achieving a uniform upstream pressure distribution and minimal blade-to-blade loading variation. By allowing the optimizer to modify only blade camber and stagger angle it is possible to arrive at a cascade geometry that minimizes the variation of blade loading around the cascade. However, this geometry does not fully minimize the upstream pressure distortion and the fan forced vibration caused by the downstream pylon. In order to fully eliminate the upstream pressure distortion one must allow for some form of rigid body translation in the optimization process. All cases that allowed either axial or tangential translation of the blades, or indeed both, resulted in fully minimized upstream pressure distortion and minimized blade-to-blade loading variation. As such, it can be understood that 
a designer, in order to fully satisfy the objective of minimized fan forcing while maintaining equally loaded blades, must have the freedom to explore more non-conventional cascades, with either non-uniform solidity or varying axial position.

\subsection{Validation with CFD}

The result of the optimizations presented above were a series of cascades that demonstrated significantly more uniform pressure fields for the upstream rotor as well as reduced blade loading variation around the guide vane cascade. In order to validate the results, the baseline and optimized geometries of the CSAT case were evaluated using high fidelity RANS CFD with an in-house code. The code used was initially described by di Mare et al. [21], was validated by Carnevale et al. [22] and has been used by Carnevale et al. [23] for a computational study of intake

lip separation, by Wang et al. [24] for the simulation of an entire virtual gas turbine, and by Carnevale et al. [25] to study low frequency distortions due to off-design operation of a large civil aero-engine intake.

Solution of the Reynolds-averaged Navier-Stokes equations is through a cell-centered finite volume approach using a pseudotime marching method on unstructured girds. The unsteady RANS equations can be written as:

$$
\begin{gathered}
\frac{\partial \bar{\rho}}{\partial t}+\frac{\partial\left(\bar{\rho} \widetilde{v_{i}}\right)}{\partial x_{j}}=0 \\
\frac{\partial\left(\bar{\rho} \widetilde{v_{i}}\right)}{\partial t}+\frac{\partial\left(\bar{\rho} \widetilde{v_{i}} \widetilde{v_{j}}\right)}{\partial x_{j}}=-\frac{\partial \bar{p}}{\partial x_{i}}+\frac{\partial}{\partial x_{j}}\left(\widetilde{\tau_{i j}}+\tau_{i j}^{t}\right) \\
\frac{\partial(\bar{\rho} \widetilde{E})}{\partial t}+\frac{\partial\left(\bar{\rho} \widetilde{v_{j}} \widetilde{H}\right)}{\partial x_{j}}=\frac{\partial}{\partial x_{j}}\left(\kappa \frac{\partial \widetilde{T}}{\partial x_{j}}+\widetilde{v_{i}}\left(\widetilde{\tau_{i j}}+\tau_{i j}^{t}\right)\right)
\end{gathered}
$$

where a tilde represents Favre averaging and an overbar denotes Reynolds averaging. Furthermore,

$$
\widetilde{\tau_{i j}}=2 \mu\left(\widetilde{S_{i j}}-\frac{1}{3} \frac{\partial \widetilde{v_{k}}}{\partial x_{k}} \delta_{i j}\right)
$$




$$
\begin{gathered}
\bar{p}=(\gamma-1) \bar{\rho}\left(\widetilde{E}-\frac{1}{2} \widetilde{u_{j}} \widetilde{u_{j}}\right) \\
\kappa \frac{\partial T}{\partial x_{j}}=\frac{\gamma}{\gamma-1} \frac{\mu}{\operatorname{Pr}} \frac{\partial}{\partial x_{j}}\left(\frac{\bar{p}}{\bar{\rho}}\right)
\end{gathered}
$$

The solver considers the working fluid to be air and to be a calorifically perfect gas. As such, the ratio of specific heats $(\gamma)$ and the Prandtl number $(P r)$ are considered constants defined as $\gamma=1.4$ and $P r=0.72$. The dynamic viscosity $(\mu)$ is calculated using Sutherland's formula. The Boussinesq approximation is used to evaluate the Reynolds stress $\left(\tau_{i j}^{t}\right)$ as

$$
\tau_{i j}^{t}=2 \mu_{t}\left(\widetilde{S_{i j}}-\frac{1}{3} \frac{\partial \widetilde{v_{k}}}{\partial x_{k}} \delta_{i j}\right)
$$

Numerical fluxes are calculated using a total variation diminishing scheme with a van Albada limiter [26]. Gradients are evaluated using a weighted least squares method. The inviscid fluxes are evaluated using Roe's flux vector difference splitting [27]. Convergence is obtained using Jacobi iterations and the low Reynolds number implementation of the $k-\omega$ turbulence model is used [28]. The solver has been optimized to run efficiently on modern multicore and manycore architectures [29, 30].

A hybrid unstructured mesh has been used for the CFD simulations of the cascades. The mesh was generated using the methods of Wang \& di Mare [31]. A view of the typical mesh used in the CFD simulations is provided in Fig. 8. The main computations presented below were run on a modern HPC using 12 cores. Convergence was typically achieved within 14 hours of elapsed real time.

A grid convergence study was performed using three grids, one with a first cell height in the boundary layer one half that of the grid shown in Fig. 8, termed the 'fine' grid, the 'medium' grid shown in Fig. 8, and a 'coarse' grid with twice the first cell height as the medium grid. 


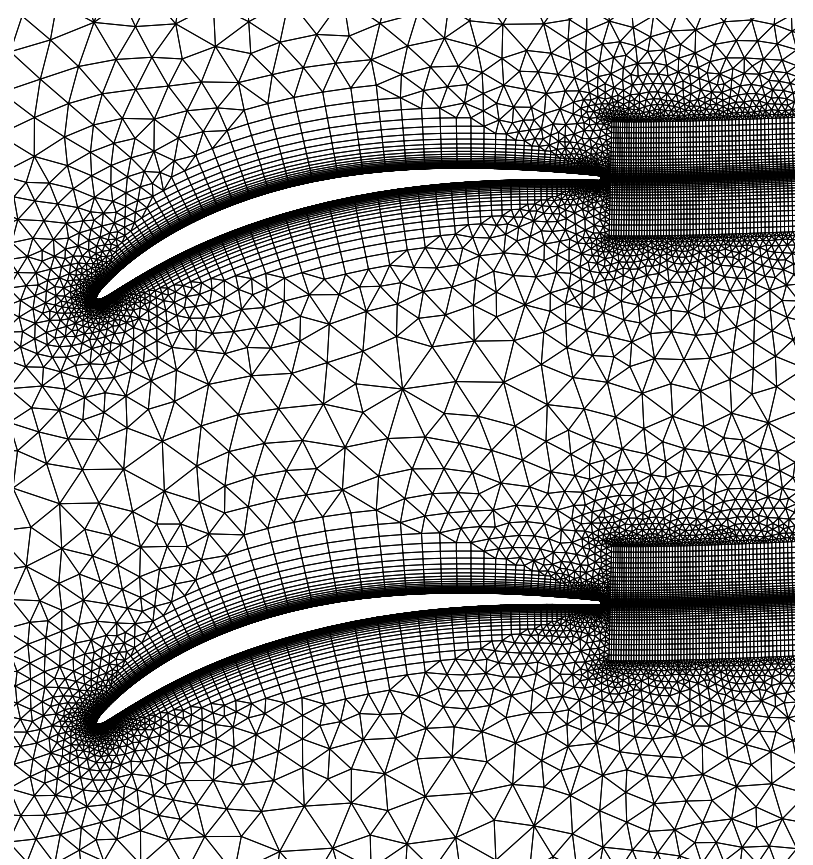

Figure 8: View of hybrid mesh near blades and pylon leading edge typical of that used for CFD simulation of baseline and optimized cascades. 
The baseline cascade was run on the three computational girds and boundary layer profiles along the chord of a lightly and highly loaded blade were recorded. Figure 9 shows the boundary layer velocity in wall coordinates for the three computational grids at 10\%, 20\%, 50\%, $80 \%$, and $90 \%$ of the blade chord. The log law is also plotted for each profile. There is a discrepancy between the log law profile and the numerical boundary layer profiles. The cause of the discrepancy is the adverse pressure gradient associated with the diffusion of the guide vanes. The log law profile is for a boundary layer on a flat plate. As the profiles are taken along a convex surface in a pressure field with an adverse gradient, some deviation is expected.

The boundary layer profiles for the coarse grid deviate some from the medium and fine results. The difference is most noticeable in the plot for $50 \%$ chord for the lightly loaded blade (top middle graph), though there is still a noticeable difference at $80 \%$ and $90 \%$ chord for the same blade. There is no noticeable difference between the results of the medium and fine grids.

Based on the results of the grid convergence study, the medium mesh was selected and a mesh with equivalent parameters was used for the optimized geometry.

Contour lines of pressure coefficient are plotted from the CFD results in Fig. 10. Isobars are plotted for the baseline geometry (top) and the optimized geometry (bottom). Several features are of note. Firstly, the contours for the baseline geometry show a clear variation of the pressure coefficient in the tangential direction upstream of the cascade. This variation is absent ahead of the optimized cascade. Secondly, the areas of the regions of peak suction vary around the baseline cascade quite significantly. This is most evident by comparing the contours by the suction surface near the leading edges of the blade by markers ' $\mathrm{A}$ ' and 'B'. In the optimized case, the areas of peak suction are noticeably more similar between blades by markers 'A' and 'B'. The pressure coefficient contours presented in Fig. 10 demonstrate qualitatively that the optimized geometry reduces the upstream pressure distortion and blade-to-blade loading variation, even when viscous effects are considered.

To better compare the potential method and the CFD results the change in the pressure coefficient $\left(\Delta C_{p}\right)$ at a 


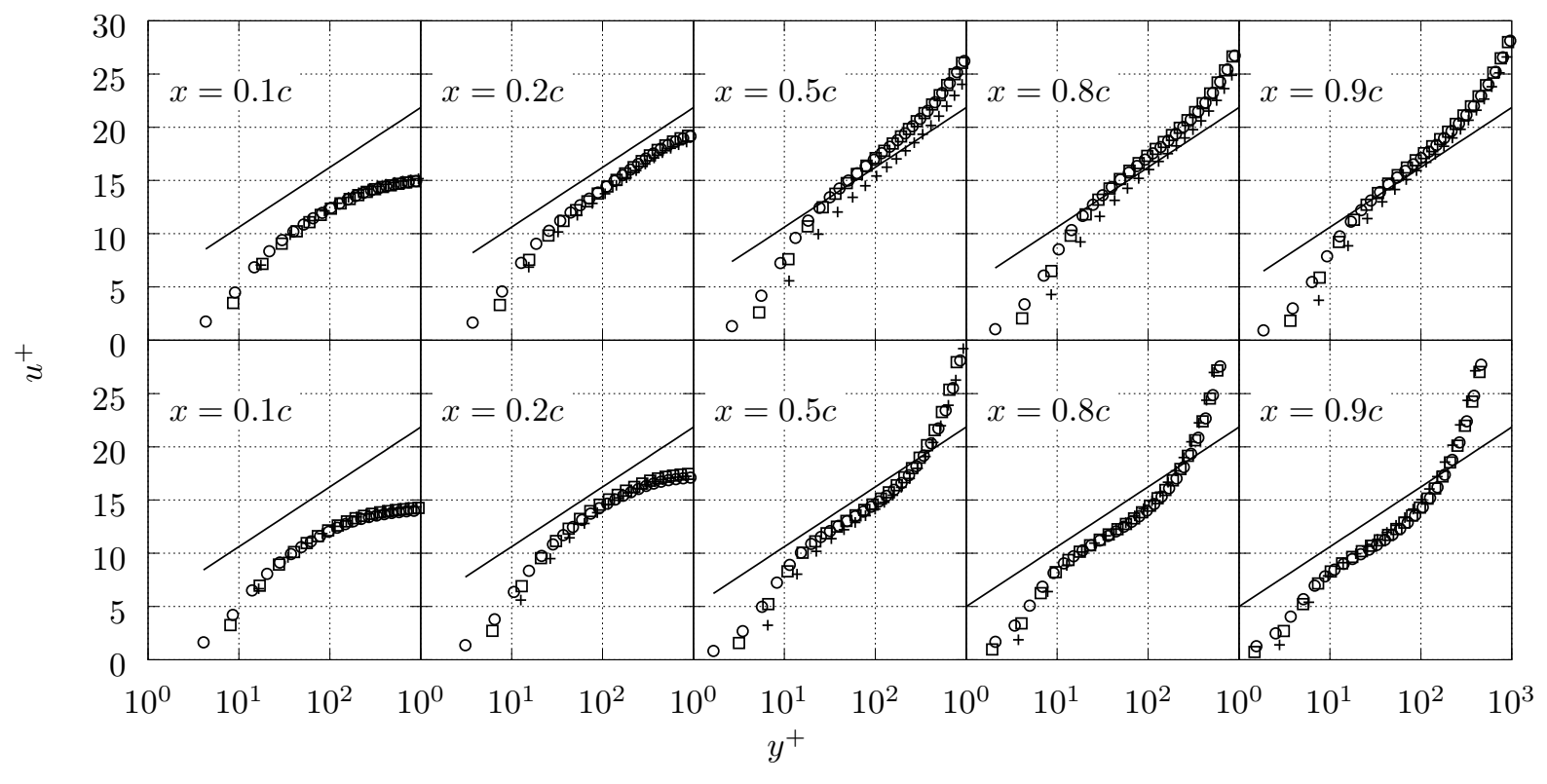

Coarse + Medium $\quad \square \quad$ Fine $\circ$ Log law

Figure 9: Boundary layer profiles for grid dependence analysis on suction surface of lightly loaded blade (top) and highly loaded blade (bottom) 


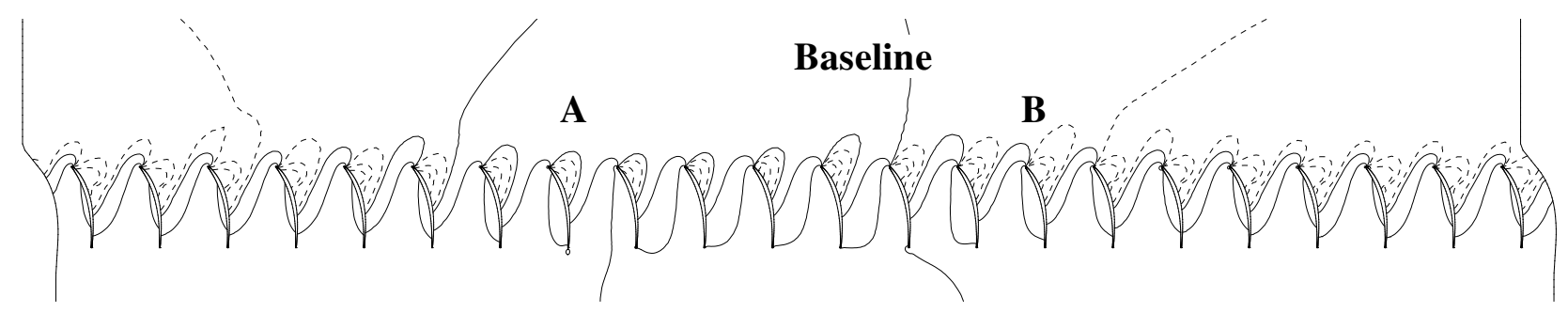

\section{Optimised}

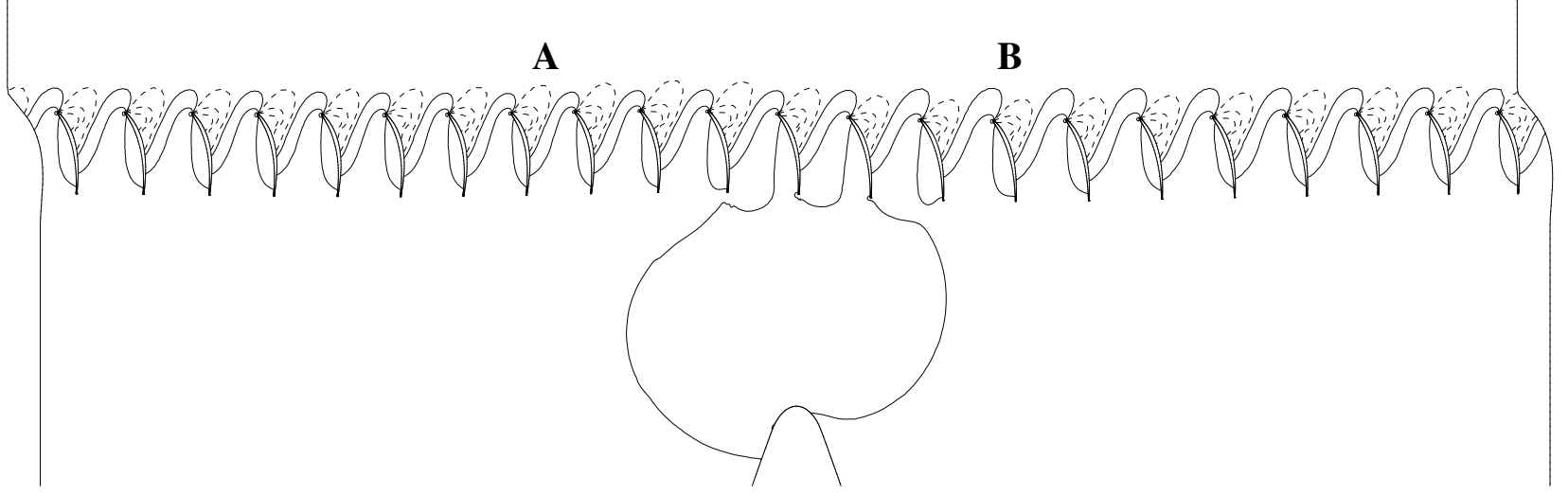

Figure 10: Contours of pressure coefficient. Levels at $\pm 0.05, \pm 0.3$, and \pm 0.6 . Negative values shown by dashed lines. Baseline geometry is at top of figure, optimized geometry is at bottom. 


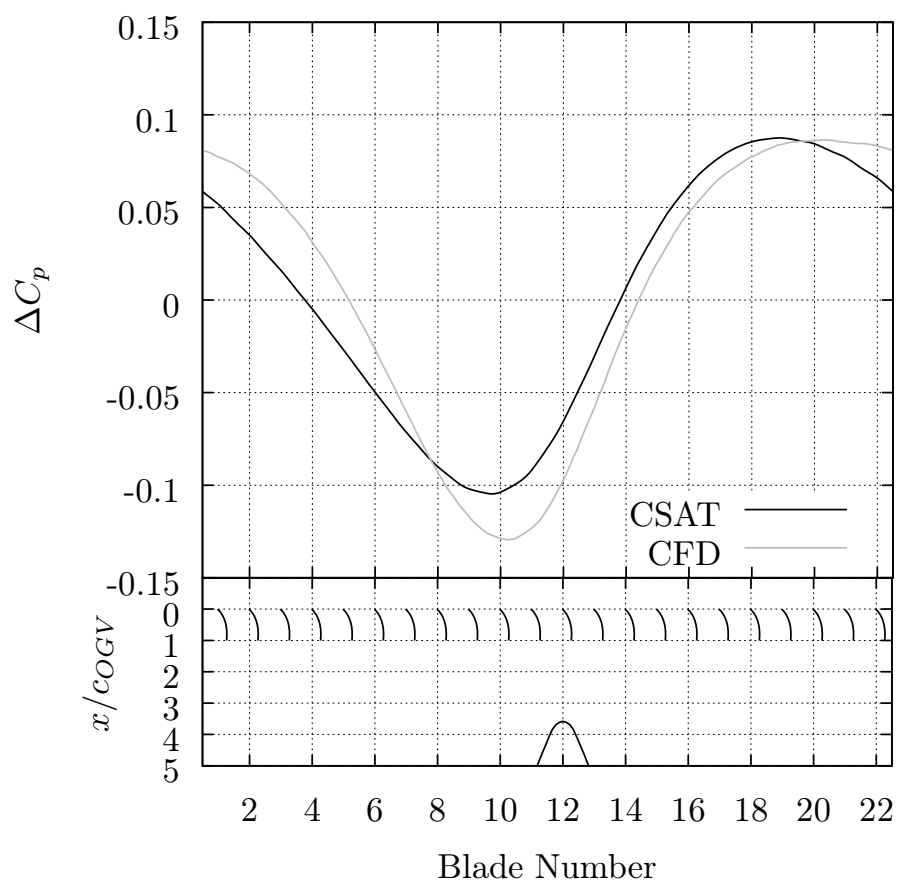

Figure 11: Change in upstream pressure coefficient from optimization for potential method and CFD.

location 1 chord upstream from the baseline cascade leading edge is plotted in Fig. 11. Here, $\Delta C_{p}=C_{p_{\text {opt }}}-C_{p_{\text {base }}}$ where $C_{p_{\text {opt }}}$ is the upstream pressure coefficient for the optimized case and $C_{p_{\text {base }}}$ is the upstream pressure coefficient for the baseline case. Figure 11 demonstrates good agreement in the change in upstream pressure coefficients between the results of the potential method and the CFD.

Further comparison between the CFD and the potential solution is provided in Fig. 12, where the difference in the blade loading before and after optimization is presented for both the CFD and potential method results. For most blades a local extremum exists in the first quarter chord of the CFD results which is caused by the presence of a shock on the blade suction surface, the location of which is modified for the optimized blades. The change in location of the shock results in a spike in the graphs. The potential method does not capture the shock formation 
and so cannot recreate this feature. Apart from this difference, the potential method results match well with the CFD results in regions away from the shock.

The total pressure coefficient, defined as $\frac{P_{0}-P_{0}}{P_{0}}$, at a position $x / c_{O G V}=2$ is plotted in Figure 13 . The total pressure coefficient provides an insight into the total pressure loss of the wakes of the guide vanes. The total pressure pressure coefficient is affected by the the peak Mach number and incidence angle of the blades, though is not a direct measure of either. For the baseline cascade, the results of the CFD show a noticeable variation in total pressure loss from each of the wakes around the cascade. In addition to more uniform wakes around the cascade, the amplitude of the total pressure loss for the optimized cascade is noticeably less than the baseline case, with most wakes having a smaller total pressure coefficient amplitude. Although not targeted, total pressure loss of the guide vane cascade has been reduced as a byproduct of fulfilling the targets of uniform blade-to-blade loading and uniform upstream pressure distribution. Loss can often be represented as a function of peak Mach number and incidence. As such, the current optimization strategy of minimizing blade-to-blade loading helps to ensure that all blades have similar values of peak Mach number and incidence, which in turn results in reduced total pressure loss through the cascade.

\section{Conclusions}

A method of optimizing guide vane cascades, based on a surface singularity method, has been presented. The optimization approach uses a least squares solution to apply geometrical changes. Geometry changes available to the optimizer modify the camber and stagger of each blade, as well as blade axial and tangential location. By employing a least squares approach informed by linearized gradients, the required geometry modifications are quickly identified and an iterative method is avoided, significantly reducing optimization time. Changes in blade axial and tangential position, through solid body translations, were shown to be able to provide significant reductions in upstream pressure distortion. It was found that in order to simultaneously satisfy the uniform upstream pressure distribution target and the uniform blade-to-blade loading target the blade location must be available as a design parameter. Limiting 


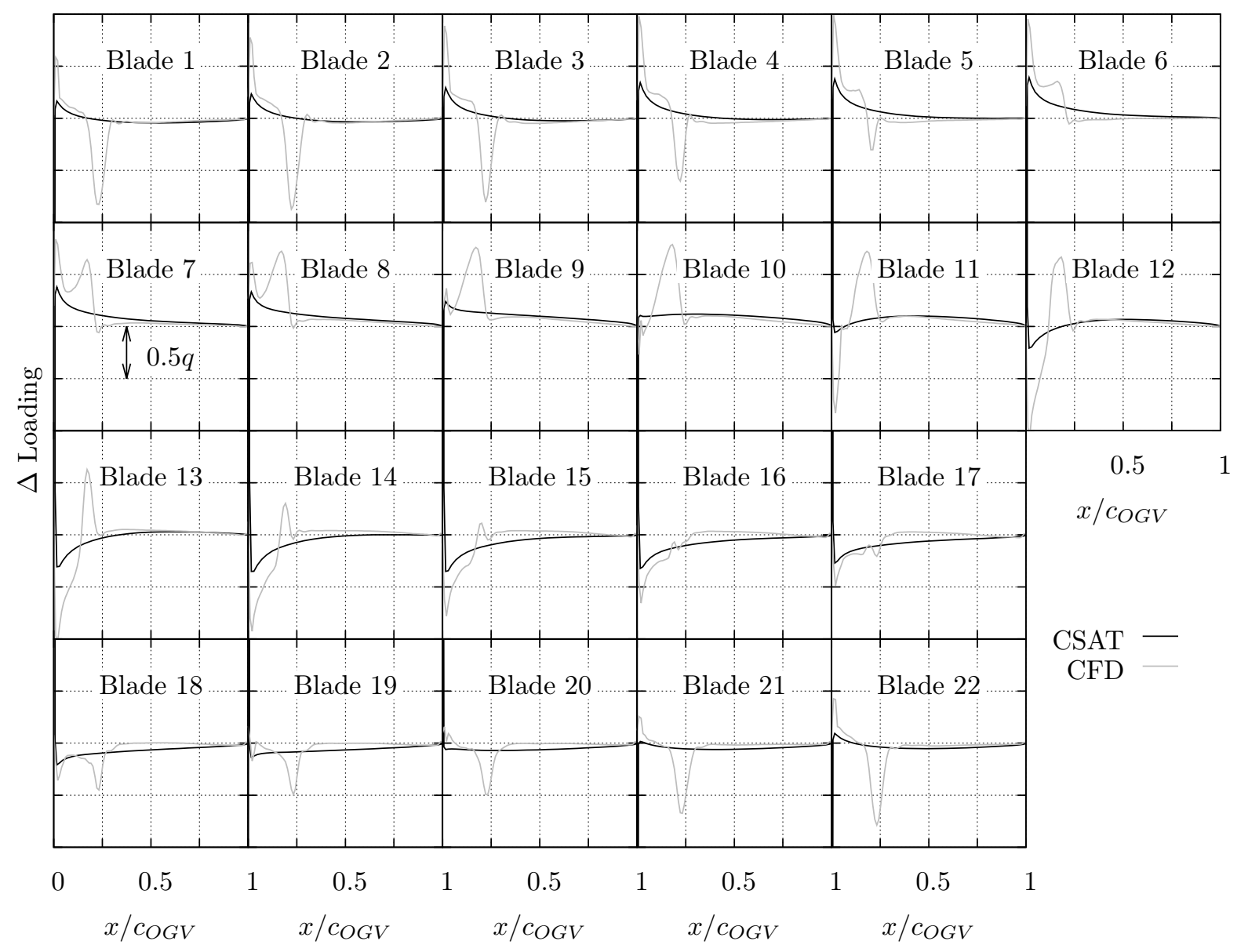

Figure 12: Comparison of change in blade loadings after optimization for CSAT case and CFD results. 


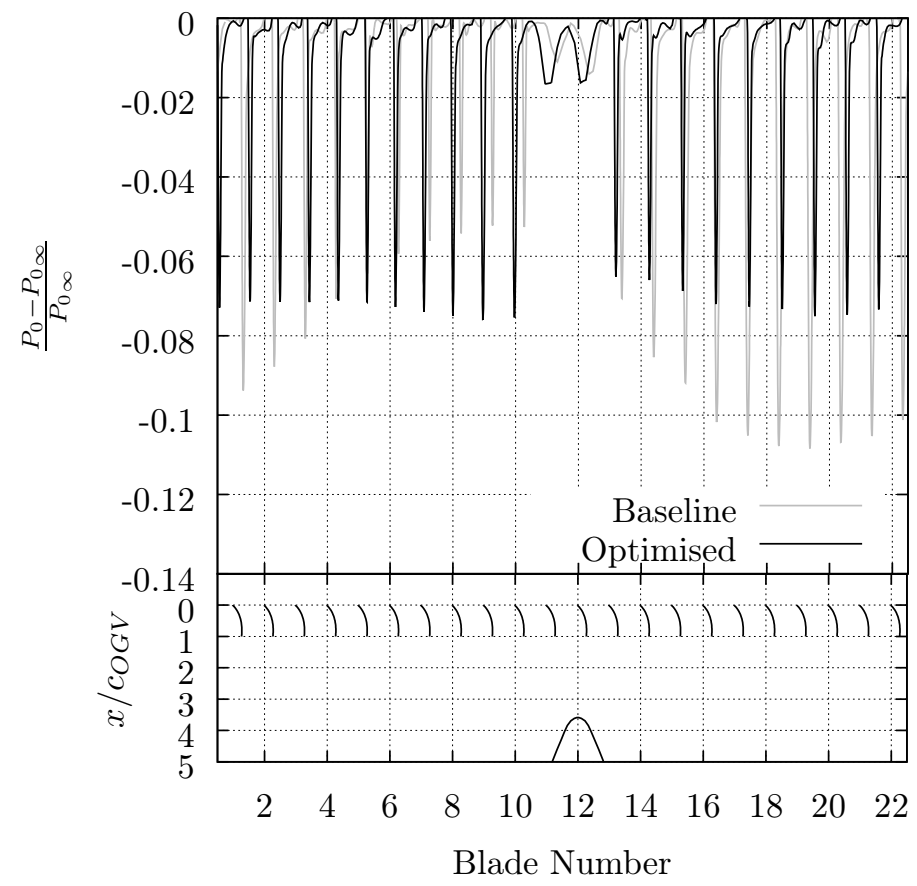

Figure 13: Downstream total pressure coefficient for baseline and optimized cascades.

the optimization to re-staggering and re-cambering only was found to yield a geometry that cannot fulfil both design targets.

The results of the potential model were compared with results from high fidelity CFD. Comparison of the changes in upstream pressure field and blade loading from baseline to optimized geometries agreed well between the potential method and the CFD results. While the CFD demonstrated the presence of shocks within the cascade, the changes in loading distribution agreed well with the potential method. Additionally, the total pressure loss of the cascade was found to be reduced for the optimized cascade. The comparison between the potential method and CFD results suggests that changes in the flowfield can be accurately targeted by the optimization technique. As the optimization problem tackled in this work is that of a cascade and a pylon, where the long range potentials are the dominant 
influencing factors, the subtle details of the flowfield around each each blade can be safely neglected in the early design stage. These encouraging results demonstrate that optimization of a guide vane cascade to mask a downstream disturbance can be performed accurately with a potential tool, providing an excellent initial design. As the complexity of the proposed initial designs increases, the flowfield may become increasingly more three-dimensional in nature. In this case a three-dimensional tool may become necessary for optimization. A possible method of three-dimensional optimization can be found in [32].

One common criticism of inviscid methods such as that employed in this work is the possibility of arriving at geometries that may not be able to turn the viscous flow without separation. By employing an optimization strategy that aims to achieve a known loading distribution on each blade, the possibility of requiring a blade to perform too much turning is avoided. Furthermore, this approach avoids the need for any constraints to the geometric deformation during optimization as only realistic geometries will be able to satisfy the target loading distribution. Finally, by employing a least squares approach to optimization a costly iterative method is avoided, and designs can be quickly optimized, enhancing the value of the tool in the early design stage. For the current work, the CS, CSA, and CST optimizations each took about 270 seconds to complete and the CSAT optimization took approximately 360 seconds to complete. Each CFD simulation took about 14 hours to converge. Assuming that a linearized CFD based optimization method takes about twice as long as a single run to perform a gradient based optimization, it is expected that a similar CFD based optimization would take approximately 28 hours for each case. Based on this, the current approach is approximately 280 times faster than a linearized CFD based optimization.

\section{Acknowledgements}

This work was carried out as part of the NADIT programme, sponsored by Rolls-Royce plc and the UK Technology Strategy Board with grant number TSB 101368.

The authors gratefully acknowledge Rolls-Royce plc and the TSB for funding this work and granting permission 
for its publication.

\section{References}

[1] Hemsworth, M. C., "Development and Experiences of the First High Bypass Ratio Engine - TF39" 11th AngloAmerican Aeronautical Conference, London, 1969.

[2] Rubbert, P., Boctor, M., Cowan, S. and LaPrete, R., "Concept and Design of Stators Tailored to Shield a Fan from Pressure Disturbances Arising in the Downstream Fan Ducts" 10th Aerospace Sciences Meeting, Paper No. 72-84, San Diego, 1972.

doi:10.2514/6.1972-84

[3] Barber, T. J., and Weingold, H. D., "Vibratory Forcing Functions Produced by Nonuniform Cascades" Journal of Engineering for Power, Vol. 100, No. 1, pp 82-88. 1978.

doi:10.1115/1.3446330

[4] Yokoi, S., Nagano, S. and Kakehi, Y., "Reduction of Strut Induced Rotor Blade Vibration with the Modified Stator Setting Angles" Fifth International Symposium on Airbreathing Engines, Bangalore, 1981.

[5] Cerri, G. and O’Brien Jr., W. F., "Sensitivity Analysis and Optimum Design Method for Reduced Rotor-StatorStrut Flow Interaction" Journal of Turbomachinery, Vol. 111, No. 4, pp 401-408, 1989.

doi: $10.1115 / 1.3262287$

[6] O’Brien, W. F., Reimers, S. L. and Richardson, S. M., "Interaction of Fan Rotor Flow with Downstream Struts" AIAA 8th Aeroacoustics Conference, Paper No. AIAA-83-0682, Atlanta, 1983.

doi:10.2514/6.1983-682 
[7] Ng, W. F., O’Brien, W. F., and Olsen, T. L., "Experimental Investigation of Unsteady Fan Flow Interaction with Downstream Struts" Journal of Propulsion and Power Vol. 3, No. 2, pp. 157-163. 1987. doi: $10.2514 / 3.22968$

[8] O'Brien, W. F., Ng, W. F., and Richardson, S. M., "Calculation of Unsteady Fan Rotor Response Caused by Downstream Flow Distortions" Journal of Propulsion and Power, Vol. 1, No. 6, pp 464-469, 1985. doi: $10.2514 / 3.22829$

[9] Holewa, A., Weckmüller, C., and Guérin, S. "Impact of Bypass Duct Bifurcations on Fan Noise" Journal of Propulsion and Power, Vol. 30, No. 1, pp 143-152, 2014.

doi:10.2514/1.B34957

[10] Giacché, D., Xu, L., and Coupland, J. "Optimization of Bypass Outlet Guide Vane for Low Interaction Noise" AIAA Journal, Vol. 52, No. 6, pp 1145-1158, 2014.

doi:10.2514/1.J052003

[11] Kodama, H., "Performance of Axial Compressor With Nonuniform Exit Static Pressure" Journal of Turbomachinery, Vol. 108, No. 1, pp 76-81, 1986.

doi:10.1115/1.3262027

[12] Kodama, H. and Nagano, S., "Potential Pressure Field by Stator/Downstream Strut Interaction" Journal of Turbomachinery, Vol. 111, No. 2, pp 197-203, 1989.

doi:10.1115/1.3262256

[13] Parry, A. B., "Optimisation of Bypass Fan Outlet Guide Vanes" International Gas Turbine and Aeroengine Congress and Exposition, Paper No. 96-GT-433, Birmingham, UK, 1996.

doi:10.1115/96-GT-433 
[14] Cerri, G., Boatto, P., O’Brien Jr., W.F. and Sorrenti, A., "Optimization of Rotor-Stator-Strut Potential Flow Interaction Including Rotor Feedback Effects" ASME 1994 International Gas Turbine and Aeroengine Congress and Exposition, Paper No. 94-GT-274, The Hague, 1994.

doi:10.1115/94-GT-274

[15] Parry, A. B., and Bailey, R. H., "The Use of Cyclic Variations in Strut Stagger to Reduce Coupled Blade-VaneStrut-Pylon Interaction and System Losses" International Gas Turbine and Aeroengine Congress and Exposition, Paper No. 97-GT-470, Orlando, 1997.

doi:10.1115/97-GT-470

[16] Chiang, H.-W. D. and Turner, M. G., "Compressor Blade Forced Response Due to Downstream Vane-Strut Potential Interaction" Journal of Turbomachinery, Vol. 118, No. 1, pp 134-142, 1996.

doi:10.1115/1.2836594

[17] Shahpar, S., Giacche, D. and Lapworth, L., "Multi-Objective Design and Optimization of Bypass Outlet-Guide Vanes" ASME Turbomachinery Technical Conference 83 Exposition 2003, Paper No. GT2003-38700, Atlanta, 2003. doi:10.1115/GT2003-38700

[18] Enoki, T., Kodama, H. and Kusuda, S., "Investigation of Fan Rotor Interaction with Pressure Disturbance Produced by Downstream Pylon" ASME Turbomachinery Technical Conference \& Exposition 2013, Paper No. GT2013-94772, San Antonio, 2013.

doi:10.1115/GT2013-94772

[19] Rife, M. E., Parry, A. B. and di Mare, L., "Fan Forced Response Reduction Through Bypass Outlet Guide Vane Optimisation" International Symposium on Unsteady Aerodynamics, Aeroacoustics and Aeroelasticity of Turbomachines, Paper No. I14-S4-2, Stockholm, 2015. 
[20] Rife, M. E., Barbarossa, F., Parry, A. B., Green, J. S. and di Mare, L., "Minimisation of Ducted Flow NonUniformity Caused by Downstream Blockages" ASME Turbomachinery Technical Conference \& Exposition 2016, Paper No. GT2016-56199, Seoul, 2016.

doi:10.1115/GT2016-56199

[21] di Mare, L., Kulkarni, D. Y., Wang, F., Romanov, A., Ramar, P. R. and Zachariadis, Z. I., "Virtual Gas Turbines: Geometry and Conceptual Description" ASME Turbomachinery Technical Conference 83 Exposition 2011, Paper No. GT2011-46437, Vancouver, 2011.

doi:10.1115/GT2011-46437

[22] Carnevale, M., Green, J. S. and di Mare, L., "Numerical Studies Into Intake Flow for Fan Forcing Assessment" ASME Turbomachinery Technical Conference Es Exposition 2014, Paper No. GT2014-25772, Düsseldorf, 2014. doi:10.1115/GT2014-25772

[23] Carnevale, M., Wang, F., Green, J. S. and di Mare, L., "Lip Stall Suppression in Powered Intakes" Journal of Propulsion and Power, Vol. 32, No. 1, pp 161-170, 2016.

doi: $10.2514 / 1 . B 35811$

[24] Wang, F., Carnevale, M., Lu, G., di Mare, L. and Kulkarni, D., "Virtual Gas Turbine: Pre-Processing and Numerical Simulations" ASME Turbomachinery Technical Conference $\&$ Exposition 2016, Paper No. GT201656227, Seoul, 2016.

doi:10.1115/GT2016-56227

[25] Carnevale, M., Wang, F., and di Mare, L., "Low Frequency Distortion in Civil Aero-Engine Intake" Journal of Engineering for Gas Turbines and Power, Vol. 139, No. 4, pp 041203-1-041203-12. doi:10.1115/1.4034600

[26] Hirsch, C., Numerical Computation of Internal and External Flows, Vol. 2, Wiley, Chichester, 1990. 
[27] Roe, P. L., "Approximate Riemann Solvers, Parameter Vectors and Difference Schemes" Journal of Computational Physics, Vol. 43, No. 2, pp 357-372, 1981.

doi: $10.1016 / 0021-9991(81) 90128-5$

[28] Wilcox, D. C. 1988, "Re-Assessment of the Scale-Determining Equation for Advanced Turbulence Models" AIAA Journal. Vol. 26, No. 11, pp 1299-1310, 1988.

doi: $10.2514 / 3.10041$

[29] Hadade, I., di Mare, L. "Exploiting SIMD and Thread-Level Parallelism in Multiblock CFD" Proceedings of the 29th International Conference on Supercomputing, Leipzig, 2014, pp 410-419.

doi:10.1007/978-3-319-07518-1_26

[30] Hadade, I., di Mare, L. "Modern Multicore and Manycore Architectures: Modelling, Optimisation, and Benchmarking a Multiblock CFD Code" Computer Physics Communications, Vol. 205, pp 32-47, 2016.

doi:10.1016/j.cpc.2016.04.006

[31] Wang, F., di Mare, L. "Hybrid Meshing Using Constrained Delaunay Triangulation for Viscous Flow Simulations" International Journal for Numerical Methods in Engineering, Vol. 108, No. 13, pp. 1667-1685, 2016.

doi: $10.1002 /$ nme.5272

[32] Barbarossa, F., Rife, M. E., Carnevale, M., Parry, A. B., Green, J. S., di Mare, L. "Fast Optimisation of a Three-Dimensional Bypass System Using a New Aerodynamic Design Method" ASME Turbomachinery Technical Conference ES Exposition 2016, Paper No. GT2017-63324, Charlotte, 2017.

doi:10.1115/GT2017-63324

\section{Appendix: Flow Field Solver Details}

The velocity field of an infinite row of vortices can be evaluated at any location, $\boldsymbol{z}$, within the complex plane as 


$$
\boldsymbol{w}(\boldsymbol{z})=\frac{\gamma i}{2 \sigma} \operatorname{coth}\left(\frac{\pi}{\sigma}\left(\boldsymbol{z}-\boldsymbol{z}_{0}\right)\right)
$$

where $\boldsymbol{w}(z)$ is the complex velocity at $\boldsymbol{z}$. The complex velocity is of the form $\boldsymbol{w}(\boldsymbol{z})=u-i v$ where $u$ and $v$ are the real and imaginary components of the velocity, respectively. $\gamma$ is the vortex circulation of the singularity, $\sigma$ is the pitch of the cascade, and $\boldsymbol{z}_{0}$ is the location of the vortex. The influence coefficient matrix, $\boldsymbol{A}$ is then generated, with elements $a_{i j}$, representing the induced velocity at panel $j$ caused by a vortex on panel $i$. The influence coefficient is calculated as

$$
a_{i j}=\boldsymbol{w}_{i} \cdot \boldsymbol{n}_{j}
$$

where $\boldsymbol{n}_{j}$ is the normal vector to panel $j$.

The vortex strength is obtained by solving a linear system of equations in which the right hand side is the dot product of the upstream velocity at infinity (inlet boundary condition) and the normal to the panel, i.e.

$$
R H S_{i}=-\boldsymbol{q}_{\infty} \cdot \boldsymbol{n}_{i}
$$

where $q_{\infty}$ is the upstream velocity.

Then the equation for panel $i$ can be expressed as the sum of the product of influences and vortex strengths from all elements

$$
\sum_{j} a_{i j} \cdot \gamma_{i}=R H S_{i}
$$

The complete system of equations for $n$ panels of unknown vortex strengths, $\gamma_{n}$ then has the form 


$$
\left[\begin{array}{cccc}
a_{11} & a_{12} & \cdots & a_{1 n} \\
a_{21} & a_{22} & \cdots & a_{2 n} \\
\vdots & \vdots & \ddots & \vdots \\
a_{n 1} & a_{n 2} & \cdots & a_{n n}
\end{array}\right]\left[\begin{array}{c}
\gamma_{1} \\
\gamma_{2} \\
\vdots \\
\gamma_{n}
\end{array}\right]=\left[\begin{array}{c}
R H S_{1} \\
R H S_{2} \\
\vdots \\
R H S_{n}
\end{array}\right]
$$

Solution of this system of equations then yields the vortex strengths for each panel.

For bodies with significant thickness, such as the pylon, the thickness distribution cannot be neglected and must be included in the problem. Like the thin blades, thick bodies are discretized with straight line panels. Following the method of Cerri [5] each panel contains a constant source and constant vortex distribution. Then the thick bodies can be handled in a similar way to the thin bodies. The velocity at any location within the complex plane, $\boldsymbol{w}(\boldsymbol{z})$, can be evaluated as

$$
\boldsymbol{w}(\boldsymbol{z})=\ln \left[\frac{\sinh \left(\frac{\pi}{\sigma}(y-a)\right)}{\sinh \left(\frac{\pi}{\sigma}(y+a)\right)}\right]
$$

where $y$ is the tangential separation between the interrogation point and the panel, and $a$ is the length of the panel.

A matrix of influence coefficients, $\boldsymbol{A}$, can be generated, taking the form

$$
\boldsymbol{A}=\left[\begin{array}{cccccc}
a_{11} & a_{12} & \cdots & a_{1 n} & \Sigma b_{1 j} & n y_{1} \\
a_{21} & a_{22} & \cdots & a_{2 n} & \Sigma b_{2 j} & n y_{2} \\
\vdots & \vdots & \ddots & \vdots & \vdots & \vdots \\
a_{n 1} & a_{n 2} & \cdots & a_{n n} & \Sigma b_{n j} & n y_{n} \\
a_{11}^{t}+a_{n 1}^{t} & a_{12}^{t}+a_{n 2}^{t} & \cdots & a_{1 n}^{t}+a_{n n}^{t} & \Sigma\left(b_{1 j}^{t}+b_{n j}^{t}\right) & l y_{1}+l y_{n} \\
0 & 0 & \cdots & 0 & s \frac{2 \pi}{\sigma} & 1
\end{array}\right]
$$

This can be coupled with a vector of boundary conditions, $\boldsymbol{g}$ 


$$
\boldsymbol{g}=\left[\begin{array}{c}
-U_{\infty} n x_{1} \\
-U_{\infty} n x_{2} \\
\vdots \\
-U_{\infty} n x_{n} \\
-U_{\infty} l x_{1}-U_{\infty} l x_{n} \\
V_{\infty}
\end{array}\right]
$$

where $a_{i j}$ is the normal velocity at $j$ caused by a unit strength source at panel $i$ and $a_{i j}^{t}$ is the tangential component of the velocity. Similarly, $b_{i j}$ is the normal velocity at $j$ caused by a unit strength vortex at panel $i$ and $b_{i j}^{t}$ is the tangential component of the velocity. $s$ is used to denote the perimeter of the thick body. Values $n y_{i}$ and $n x_{i}$ represent the tangential and axial components of the unit vector of panel $i$, respectively, and $l y_{i}$ and $l x_{i}$ denote the tangential and axial components of the distance between the end points of panel $i . U_{\infty}$ and $V_{\infty}$ are the axial and tangential components of the velocity far upstream.

By inverting $\boldsymbol{A}$ and multiplying by $\boldsymbol{g}$ the source and vortex strengths at each panel on the thick body are obtained. More complex combinations of thin and thick bodies are easily solved using one matrix of influence coefficients and a single vector of boundary conditions. 